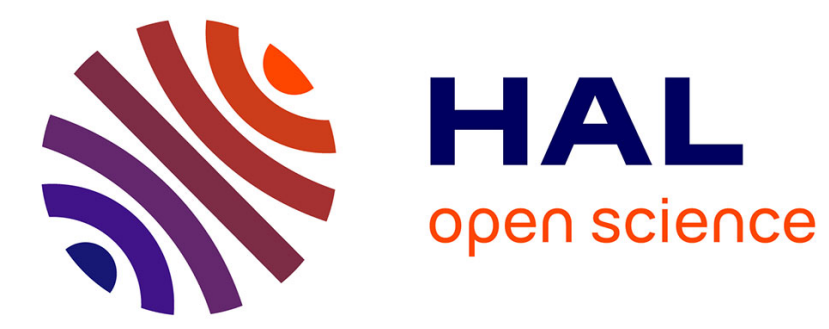

\title{
Throughput Performance in Networks with Linear Capacity Contraints
}

Thomas Bonald

\section{To cite this version:}

Thomas Bonald. Throughput Performance in Networks with Linear Capacity Contraints. CISS, 2006, Princeton, United States. hal-01277609

\section{HAL Id: hal-01277609 \\ https://hal.inria.fr/hal-01277609}

Submitted on 22 Feb 2016

HAL is a multi-disciplinary open access archive for the deposit and dissemination of scientific research documents, whether they are published or not. The documents may come from teaching and research institutions in France or abroad, or from public or private research centers.
L'archive ouverte pluridisciplinaire HAL, est destinée au dépôt et à la diffusion de documents scientifiques de niveau recherche, publiés ou non, émanant des établissements d'enseignement et de recherche français ou étrangers, des laboratoires publics ou privés. 


\title{
Throughput Performance in Networks with Linear Capacity Contraints
}

\author{
T. Bonald \\ France Telecom R\&D \\ thomas.bonald@francetelecom.com
}

\begin{abstract}
We consider a network whose resources are shared by a dynamic number of data flows according to balanced fairness. We give explicit bounds on the mean throughput that results from this stochastic resource sharing when the capacity constraints are linear. We illustrate the results on a few examples of wireline and wireless networks.
\end{abstract}

Index Terms - Flow throughput, balanced fairness.

\section{INTRODUCTION}

The design of control algorithms like routing, scheduling, congestion and power control is a central issue in communication networks. These algorithms must ensure an optimal utilisation of network resources [1], [2], [3], [4]. This in turn implies maximum stability in the sense that the number of competing flows, which varies at random as new flows arrive and other cease, remains finite as long as the traffic intensity does not exceed the network capacity [5], [6], [7], [8].

The issue of throughput degradation when traffic intensity approaches network capacity is rarely addressed, however. One reason is that usual resource allocation schemes like max-min fairness and proportional fairness do not lead to an explicit expression for the steady state distribution, which determines the typical number of competing flows [8]. Simulations tend to show that these fair allocations have very similar throughput performance provided the resource requirements of competing flows do not differ significantly. In such cases, the throughput can be accurately estimated by that of balanced fairness [8], [9]. In other cases, balanced fairness provides a good approximation of proportional fairness only while max-min fairness typically leads to much lower throughput [8].

The notion of balanced fairness was introduced as a means to get analytical expressions for the steady state distribution. A key property of balanced fairness is its insensitivity: the steady state distribution is independent of all traffic characteristics beyond the traffic intensity. Flows may arrive within sessions of arbitrary structure, with possible correlation between successive flow sizes and think-time durations. The only required assumption is that sessions arrive as a Poisson process, which is indeed satisfied in practice [10]. Performance metrics like flow throughput can then be numerically evaluated by means of a recursive algorithm [8]. The state space explosion limits the scope of such a numerical solution, however.

In this paper, we derive simple, explicit bounds on the flow throughput under balanced fairness. We only require that the network has linear capacity constraints. We first show that a number of wireline and wireless networks enter this framework. We then address stability issues and give a formal definition of flow throughput. The main result is presented in Section V and illustrated by some examples in Section VI. Section VII concludes the paper.

\section{LineAR CAPACITY CONSTRAints}

Consider a network of $L$ resources. Each resource may represent the capacity of a wireline link, the frequency band or transmission power of a wireless network, for instance. We denote by $C_{l}$ the amount of resource $l$. A random number of flows compete for access to these resources. We consider an arbitrary number of $N$ flow classes such that all flows within the same class require the same resources. Specifically, each class- $i$ flow requires an amount of resource $l$ equal to $A_{i l}$ per bit/s. Let $\phi_{i}$ be the total bit rate allocated to class- $i$ flows, which is assumed to be evenly shared by these flows. Using vectorial notations, the allocation $\phi$ must satisfy the inequality:

$$
\phi A \leq C
$$

We refer to the set of vectors $\phi$ that satisfy this inequality as the capacity set.

Wireline networks: Consider a wireline network of $L$ links. The capacity of link $l$ is $C_{l}$ (in bit/s). Let $A_{i l}=1$ if class- $i$ flows go through link $l, A_{i l}=0$ otherwise. Figures 1 and 2 give simple examples of such networks with their capacity set.
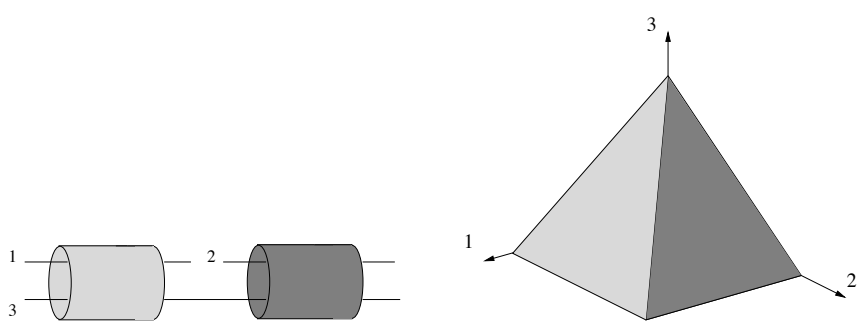

Fig. 1. A linear network and its capacity set.

Note that we do not specify the direction of the links. In particular, multicast flows may well be represented in the above framework. In Figure 1 for instance, class-3 flows may indeed correspond to multicast flows with a single source (located between the two links) and two destinations. Note also that more complex routing schemes where traffic is split over several paths enter the same framework. The capacity set is still a convex polytope. 

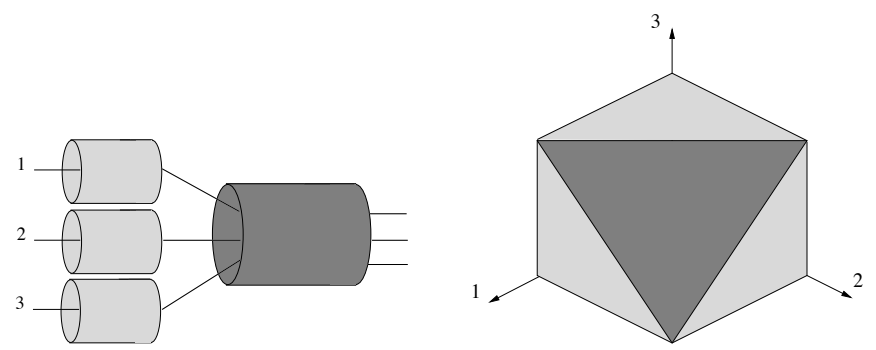

Fig. 2. A tree network and its capacity set.

Wireless networks: The capacity set of wireless networks is generally much more difficult to define. It depends both on the radio resources (frequency band, transmission power) and on the access scheme to these resources. Consider the simple case of a wireless access point that transmits data to each active mobile one at a time, using the whole frequency band and the full transmission power. A number $N$ of pre-defined modulation and coding schemes can be used by the mobiles depending on their radio conditions. Each class- $i$ flow use the modulation and coding scheme $i$ and has the bit rate $c_{i}$ when served. Thus $\phi_{i} / c_{i}$ is the fraction of time the access point serves a class- $i$ flow. The capacity constraint is then:

$$
\frac{\phi_{1}}{c_{1}}+\frac{\phi_{2}}{c_{2}}+\ldots+\frac{\phi_{N}}{c_{N}} \leq 1
$$

This is illustrated by Figure 3 for $N=3$ flow classes with $c_{1}>c_{2}>c_{3}$.
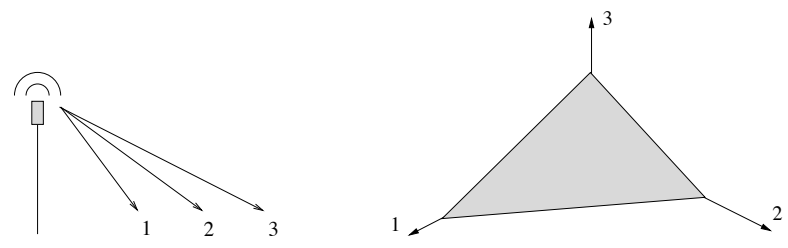

Fig. 3. A time-shared wireless access point and its capacity set.

Now consider the additional capacity constraint due to a wireline backhaul link of $C$ bit/s:

$$
\phi_{1}+\phi_{2}+\ldots+\phi_{N} \leq C .
$$

The corresponding capacity set is given in Figure 4 for $N=3$ flow classes with $c_{1}>c_{2}>C>c_{3}$.

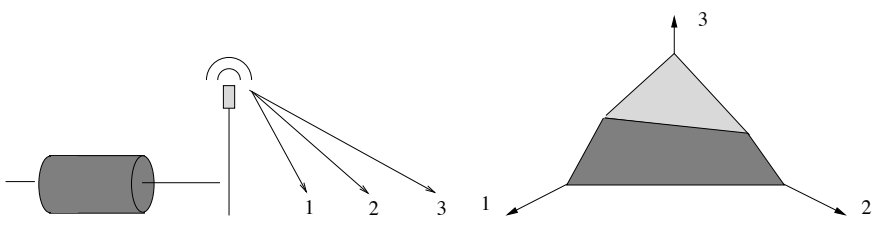

Fig. 4. A wireless access point with wireline backhaul and its capacity set.

More realistic wireless networks with several access points and various access schemes, as well as some ad-hoc networks that do not rely on any infrastructure, may also be represented as networks with linear capacity constraints [8].
Flow rate limits: In addition to the global capacity constraint (1), flows may have individual rate constraints due for instance to the speed of the user's access line in wireline networks or the capacity of the mobile in wireless networks. The impact of these additional constraints on the performance bounds is described at the end of Section V.

\section{StABILITy ISSUES}

Let $x_{i}$ be the number of class- $i$ flows. In the following, we refer to the corresponding vector $x$ as the network state. Note that the network state evolves as a stochastic process that depends both on precise traffic characteristics like the flow arrival process and the flow size distribution of each class and on the resource allocation.

Let $\rho_{i}$ be the traffic intensity of class $-i$ flows in bit/s. This is the product of the arrival rate and the mean size of class- $i$ flows. Clearly, a necessary condition for the network state to reach a finite stationary regime is that the vector $\rho$ of traffic intensities lies in the capacity set:

$$
\rho A \leq C
$$

It turns out that for usual allocations like max-min fairness and proportional fairness, as well as for balanced fairness, this condition is also sufficient, up to the critical case where the vector $\rho$ lies on the boundary of the capacity set [8]. Thus in the rest of the paper, we assume that the following componentwise strict inequality is satisfied:

$$
\rho A<C
$$

\section{Flow Throughrut}

We now introduce a throughput measure, referred to as the flow throughput, that can be derived from the stationary distribution $\pi$ of the network state. The flow throughput reflects the quality of data transfers as experienced by users in the following two senses.

Mean flow duration: The first definition is related to the mean flow duration. Specifically, the flow throughput is defined as the ratio of mean flow size to the mean flow duration. We refer to the inverse of the flow throughput, namely the ratio of the mean flow duration to the mean flow size, as the per-bit delay (in s/bit). Let $\tau_{i}$ be the per-bit delay of class- $i$ flows. Since the mean size of class- $i$ flows is equal to $\sigma_{i}$, the mean duration of class- $i$ flows is equal to $\sigma_{i} \tau_{i}$. Denote by $\bar{x}_{i}$ the number of class- $i$ flows in steady state. By Little's law, we have:

$$
\bar{x}_{i} \equiv \sum_{x} x_{i} \pi(x)=\rho_{i} \tau_{i} .
$$

We deduce the flow throughput of class $i$ :

$$
\gamma_{i}=\frac{1}{\tau_{i}}=\frac{\rho_{i}}{\bar{x}_{i}} .
$$


Mean instantaneous rate: The second definition corresponds to the mean instantanous rate as experienced by users. Recall that the instantanous rate of a class- $i$ flow is equal to $\phi_{i}(x) / x_{i}$ in any state $x$ such that $x_{i}>0$. Since the probability that a class- $i$ flow sees the network in state $x$ is proportional to $x_{i} \pi(x)$ in stationary regime, the flow throughput of class $i$ is given by:

$$
\gamma_{i}=\sum_{x: x_{i}>0} \frac{x_{i} \pi(x)}{\bar{x}_{i}} \times \frac{\phi_{i}(x)}{x_{i}}=\frac{1}{\bar{x}_{i}} \sum_{x: x_{i}>0} \pi(x) \phi_{i}(x) .
$$

Expression (3) then follows from the conservation equation:

$$
\rho_{i}=\sum_{x: x_{i}>0} \pi(x) \phi_{i}(x)
$$

\section{Performance Bounds}

We now give explicit bounds on the flow throughput when the allocation is balanced fairness [8]. Balanced fairness is uniquely defined by a positive function $\Phi$, referred to as the balance function. Specifically, the total bit rate of class- $i$ flows in state $x$ is given by:

$$
\phi_{i}(x)=\frac{\Phi\left(x-e_{i}\right)}{\Phi(x)},
$$

where $e_{i}$ denotes the unit vector with 1 in component $i$ and 0 elsewhere. We use the convention that $\Phi(x)=0$ if $x \notin \mathbb{N}^{N}$. In view of the capacity constraint (1), the balance function must satisfy the inequalities:

$$
\sum_{i} A_{i l} \frac{\Phi\left(x-e_{i}\right)}{\Phi(x)} \leq C_{l}, \quad l=1, \ldots, L .
$$

We say that resource $l$ is saturated in state $x$ if

$$
\sum_{i} A_{i l} \phi_{i}(x)=C_{l},
$$

that is if the corresponding inequality (4) is an equality. There is a unique function $\Phi$ such that in all states $x \neq 0$, at least one resource is saturated. In view of (4), this function is recursively defined by $\Phi(0)=1$ and:

$$
\Phi(x)=\max _{l} \sum_{i} \frac{A_{i l}}{C_{l}} \Phi\left(x-e_{i}\right) .
$$

This is the balance function associated with balanced fairness. Under the stability condition (2), the stationary distribution of the network state is then given by:

$$
\pi(x)=\pi(0) \times \Phi(x) \rho^{x},
$$

with the normalisation constant:

$$
\pi(0)=\left(\sum_{x} \Phi(x) \rho^{x}\right)^{-1},
$$

where we use the notation:

$$
\rho^{x} \equiv \prod_{i=1}^{N} \rho_{i}^{x_{i}} .
$$

Recall that the per-bit delay $\tau_{i}$ of class $i$ is equal to the inverse of the flow throughput $\gamma_{i}$ of class $i$. The main result of the paper is the following:
Theorem 1: Let $\varrho=\rho A$. We have:

$$
\max _{l} \frac{A_{i l}}{C_{l}-\varrho_{l}} \leq \tau_{i} \leq \max _{l} \frac{A_{i l}}{C_{l}}+\sum_{l} \frac{\varrho_{l}}{C_{l}} \frac{A_{i l}}{C_{l}-\varrho_{l}} .
$$

The upper bound is tighter than the store-and-forward bound derived in [11] in the context of wireline networks. The latter is obtained assuming flows consume network resources sequentially, in a store-and-forward way, and gives in the present context of linear capacity constraints:

$$
\tau_{i} \leq \sum_{l} \frac{A_{i l}}{C_{l}-\varrho_{l}} .
$$

In particular, the upper bound (7) is tight both in the light traffic regime $(\rho \sim 0)$ and in the heavy traffic regime ( $\rho$ close to the boundary of the capacity set), in which cases the flow throughput is determined by the most constraining resource(s). The store-and-forward bound, on the other hand, may be quite loose in the light traffic regime.

Flows with a single capacity contraint: Before proving Theorem 1 in its general form, we consider the case where class- $i$ flows are constrained by resource $l$ only, in the sense that $A_{i r}=0$ for all $r \neq l$. The bounds (7) then coincide and we have:

$$
\tau_{i}=\frac{A_{i l}}{C_{l}-\varrho_{l}} .
$$

We first prove this preliminary result, which will be useful for the proof of Theorem 1. We need the following lemma, proved in Appendix I.

Lemma 1: Assume class- $i$ flows are constrained by resource $l$ only. Then resource $l$ is saturated in any state $x$ such that $x_{i}>0$, that is:

$$
\Phi(x)=\sum_{j} \frac{A_{j l}}{C_{l}} \Phi\left(x-e_{j}\right) .
$$

In view of (6), we get for all states $x$ such that $x_{i}>0$ :

$$
\pi(x)=\sum_{j} \frac{A_{j l}}{C_{l}} \rho_{j} \pi\left(x-e_{j}\right),
$$

with the convention $\pi(x)=0$ if $x \notin \mathbb{N}^{N}$. Thus,

$$
\begin{aligned}
\bar{x}_{i} & =\sum_{x} x_{i} \pi(x) \\
& =\sum_{j} \frac{A_{j l}}{C_{l}} \rho_{j} \sum_{x} x_{i} \pi\left(x-e_{j}\right), \\
& =\sum_{j \neq i} \frac{A_{j l}}{C_{l}} \rho_{j} \sum_{x} x_{i} \pi\left(x-e_{j}\right) \\
& +\frac{A_{i l}}{C_{l}} \rho_{i} \sum_{x}\left(x_{i}-1\right) \pi\left(x-e_{i}\right) \\
& +\frac{A_{i l}}{C_{l}} \rho_{i} \sum_{x} \pi\left(x-e_{i}\right), \\
& =\sum_{j \neq i} \frac{A_{j l}}{C_{l}} \rho_{j} \bar{x}_{i}+\frac{A_{i l}}{C_{l}} \rho_{i} \bar{x}_{i}+\frac{A_{i l}}{C_{l}} \rho_{i}, \\
& =\frac{\varrho_{l}}{C_{l}} \bar{x}_{i}+\frac{A_{i l}}{C_{l}} \rho_{i} .
\end{aligned}
$$

Expression (8) then follows from (3). 
Lower bound: The proof of the lower bound (7) is similar to the proof of (8). Replacing (9) by the following inequality, valid for all resources $l$ in view of (5):

$$
\Phi(x) \leq \sum_{j} \frac{A_{j l}}{C_{l}} \Phi\left(x-e_{j}\right),
$$

we obtain:

$$
\tau_{i} \geq \frac{A_{i l}}{C_{l}-\varrho_{l}}
$$

Upper bound: To prove the upper bound, we add $L+$ 1 "virtual" classes. Virtual class 0 has the same capacity constraint as class $i$, virtual class $l$ has the same resource$l$ constraint as class $i$, for all $l=1, \ldots, L$. The original allocation vector $\phi$ and the new allocation vector $\tilde{\phi}$ associated with the virtual classes must satisfy the linear constraint:

$$
\phi A+\tilde{\phi} \tilde{A} \leq C
$$

where $\tilde{A}$ denotes the $(L+1) \times L$-dimensional matrix:

$$
\tilde{A}=\left(\begin{array}{cccc}
A_{i 1} & A_{i 2} & \ldots & A_{i L} \\
A_{i 1} & 0 & \ldots & 0 \\
0 & A_{i 2} & \ldots & 0 \\
\vdots & \vdots & \ddots & \vdots \\
0 & 0 & \ldots & A_{i L}
\end{array}\right) .
$$

We denote by $\tilde{x}$ the new network state associated with the virtual classes. The original network state is still denoted by $x$. The new balance function $\tilde{\Phi}$ associated with balanced fairness is recursively defined by $\tilde{\Phi}(0)=1$ and:

$$
\begin{aligned}
\tilde{\Phi}(x, \tilde{x}) & =\max _{l}\left\{\frac{A_{i l}}{C_{l}} \tilde{\Phi}\left(x, \tilde{x}-e_{0}\right)+\frac{A_{i l}}{C_{l}} \tilde{\Phi}\left(x, \tilde{x}-e_{l}\right)\right. \\
& \left.+\sum_{j} \frac{A_{j l}}{C_{l}} \tilde{\Phi}\left(x-e_{j}, \tilde{x}\right)\right\},
\end{aligned}
$$

with the convention $\tilde{\Phi}(x, \tilde{x})=0$ if $x \notin \mathbb{N}^{N}$ or $\tilde{x} \notin \mathbb{N}^{L+1}$. We have the following key result, proved in Appendix II.

Lemma 2: For any state $x \in \mathbb{N}^{N}$,

$$
\begin{aligned}
& \tilde{\Phi}\left(x, e_{0}\right)+\left(\sum_{l} \frac{A_{i l}}{C_{l}}\right) \tilde{\Phi}(x, 0) \\
& \quad \leq \quad\left(\max _{l} \frac{A_{i l}}{C_{l}}\right) \tilde{\Phi}(x, 0)+\sum_{l} \tilde{\Phi}\left(x, e_{l}\right) .
\end{aligned}
$$

Under the stability condition (2), there exists an $(L+1)$ dimensional vector $\tilde{\rho}$ such that the following component-wise strict inequality is satisfied:

$$
\tilde{\varrho} \equiv \rho A+\tilde{\rho} \tilde{A}<C .
$$

Let $\tilde{\tau}_{l}$ be the corresponding per-bit delay of virtual class $l$, for all $l=0,1, \ldots, L$. To prove the upper bound, we use the fact that:

$$
\lim _{\tilde{\rho} \rightarrow 0} \tilde{\tau}_{0}=\tau_{i}
$$

and, in view of (8),

$$
\forall l=1, \ldots, L, \quad \tilde{\tau}_{l}=\frac{A_{i l}}{C_{l}-\tilde{\varrho}_{l}} .
$$

In particular,

$$
\forall l=1, \ldots, L, \quad \lim _{\tilde{\rho} \rightarrow 0} \tilde{\tau}_{l}=\frac{A_{i l}}{C_{l}-\varrho_{l}} .
$$

Now it follows from (3) and (6) that:

$$
\forall l=0,1, \ldots, L, \quad \lim _{\tilde{\rho} \rightarrow 0} \tilde{\tau}_{l}=\frac{\sum_{x} \tilde{\Phi}\left(x, e_{l}\right) \rho^{x}}{\sum_{x} \tilde{\Phi}(x, 0) \rho^{x}} .
$$

Using Lemma 2, we obtain:

$$
\tau_{i}+\sum_{l} \frac{A_{i l}}{C_{l}} \leq \max _{l} \frac{A_{i l}}{C_{l}}+\sum_{l} \frac{A_{i l}}{C_{l}-\varrho_{l}},
$$

from which the upper bound (7) directly follows.

Flow rate limits: Assume that, in addition to the global capacity constraint (1), the bit rate of each class- $i$ flow cannot exceed some positive constant $a_{i}$. Using the same reasoning, we get the following bounds on the per-bit delay of class $i$ :

$$
\begin{aligned}
& \max \left\{\frac{1}{a_{i}}, \max _{l} \frac{A_{i l}}{C_{l}-\varrho_{l}}\right\} \leq \tau_{i} \\
& \leq \max \left\{\frac{1}{a_{i}}, \max _{l} \frac{A_{i l}}{C_{l}}\right\}+\sum_{l} \frac{\varrho_{l}}{C_{l}} \frac{A_{i l}}{C_{l}-\varrho_{l}} .
\end{aligned}
$$

Again, the upper bound is tighter than the store-and-forward bound derived in [11], which gives in this case:

$$
\tau_{i} \leq \frac{1}{a_{i}}+\sum_{l} \frac{A_{i l}}{C_{l}-\varrho_{l}} .
$$

\section{EXAMPLES}

This section is devoted to some examples that illustrate the tightness of the bounds derived in Section V. Specifically, we focus on the lower bound of the flow throughput (equivalently, the upper bound of the per-bit delay), which provides a conservative estimate of throughput performance.

Wireline networks: Consider the 2-link linear network of Figure 1 with unit capacity links. Class-1 flows and class-2 flows are constrained by a single resource so that, in view of (8),

$$
\gamma_{1}=1-\varrho_{1}, \quad \gamma_{2}=1-\varrho_{2} .
$$

For class-3 flows, it follows from (7) that:

$$
\gamma_{3} \geq \frac{\left(1-\varrho_{1}\right)\left(1-\varrho_{2}\right)}{1+\varrho_{1} \varrho_{2}} .
$$

Figure 5 illustrates the tightness of this bound for equal traffic intensities $\rho_{1}=\rho_{2}=\rho_{3}$. The bound is compared to the exact expression when the total traffic intensity at each link $\varrho_{1}=\varrho_{2}$ varies from 0 to 1 .

Now consider the 3-branch tree network of Figure 2. The common root link has capacity 2 and each branch link has capacity 1 . For equal traffic intensities, it follows from (7) that:

$$
\gamma_{1}=\gamma_{2}=\gamma_{3} \geq \frac{2\left(2-\varrho_{1}\right)\left(3-\varrho_{1}\right)}{12-3 \varrho_{1}-\varrho_{1}^{2}},
$$

where $\varrho_{1}=\rho_{1}+\rho_{2}+\rho_{3}$ corresponds the traffic intensity at the common root link. The results are shown in Figure 6 with respect to $\varrho_{1}$. 


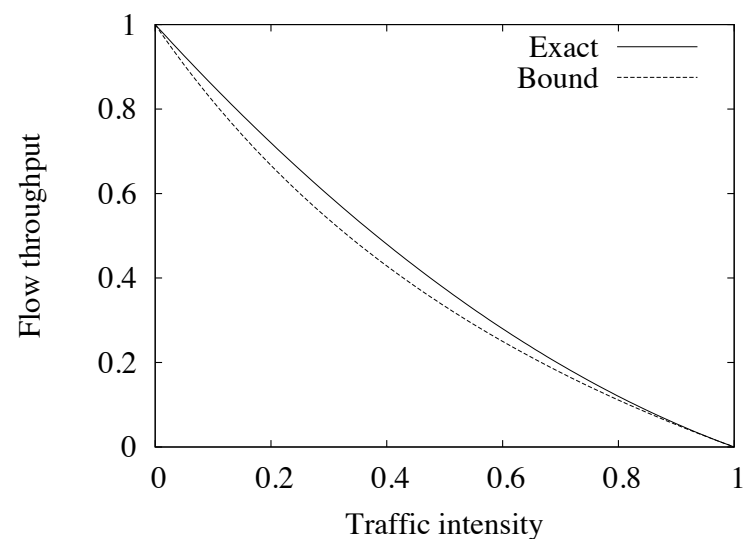

Fig. 5. Flow throughput in a 2-link linear network.

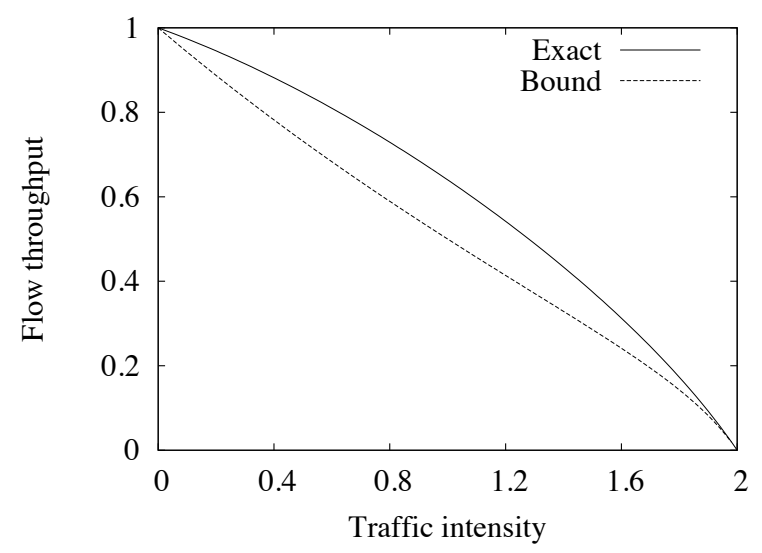

Fig. 6. Flow throughput in a 3-branch tree network.

Wireless networks: Consider the wireless access point of Figure 3. Flows are constrained by a single resource so that, in view of (8), the flow throughputs are given by:

$$
\gamma_{1}=c_{1}(1-\varrho), \quad \gamma_{2}=c_{2}(1-\varrho), \quad \gamma_{3}=c_{3}(1-\varrho),
$$

where $\varrho$ is the system load, given by:

$$
\varrho=\frac{\rho_{1}}{c_{1}}+\frac{\rho_{2}}{c_{2}}+\frac{\rho_{3}}{c_{3}} .
$$

Note that the stability condition (2) writes $\varrho<1$.

Now consider the additional constraint of a wireline backhaul link of capacity $C$, as shown in Figure 4. We get for each class $i=1,2,3$ :

$$
\gamma_{i} \geq\left(\max \left\{\frac{1}{c_{i}}, \frac{1}{C}\right\}+\frac{\varrho_{1}}{c_{i}\left(1-\varrho_{1}\right)}+\frac{\varrho_{2}}{C\left(C-\varrho_{2}\right)}\right)^{-1},
$$

where $\varrho_{1}$ and $\varrho_{2}$ correspond to the load of the wireless link and the traffic intensity on the wireline link, respectively:

$$
\varrho_{1}=\frac{\rho_{1}}{c_{1}}+\frac{\rho_{2}}{c_{2}}+\frac{\rho_{3}}{c_{3}}, \quad \varrho_{2}=\rho_{1}+\rho_{2}+\rho_{3} .
$$

The stability condition (2) writes $\varrho_{1}<1$ and $\varrho_{2}<C$. Figure 7 gives the results obtained with respect to the total traffic intensity $\varrho_{2}$ for $c_{1}=5, c_{2}=1, c_{3}=1 / 2, C=2$ and equal traffic intensities $\rho_{1}=\rho_{2}=\rho_{3}$. The stability condition reduces to $\varrho_{1}<1$ in this case, that is $\varrho_{2}<15 / 16$.

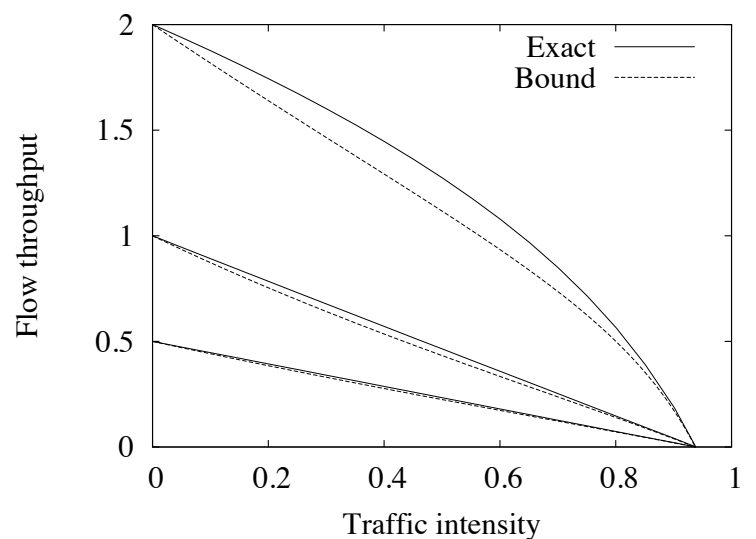

Fig. 7. Flow throughput at a wireless access point with wireline backhaul (classes 1,2,3, from top to bottom).

Flow rate limits: Finally, we consider a wireline link of $C$ bit/s shared by flows with $N=3$ different rate limits, $a_{1}, a_{2}, a_{3}<C$. It follows from (11) that for each class $i=$ $1,2,3$ :

$$
\gamma_{i} \geq\left(\frac{1}{a_{i}}+\frac{\varrho}{C(C-\varrho)}\right)^{-1}
$$

where $\varrho=\rho_{1}+\rho_{2}+\rho_{3}$ denotes the total traffic intensity. The stability condition is $\varrho<C$. Figure 8 gives the corresponding results with respect to $\varrho$ for $C=10, a_{1}=2, a_{2}=1, a_{3}=1 / 2$ and equal traffic intensities.

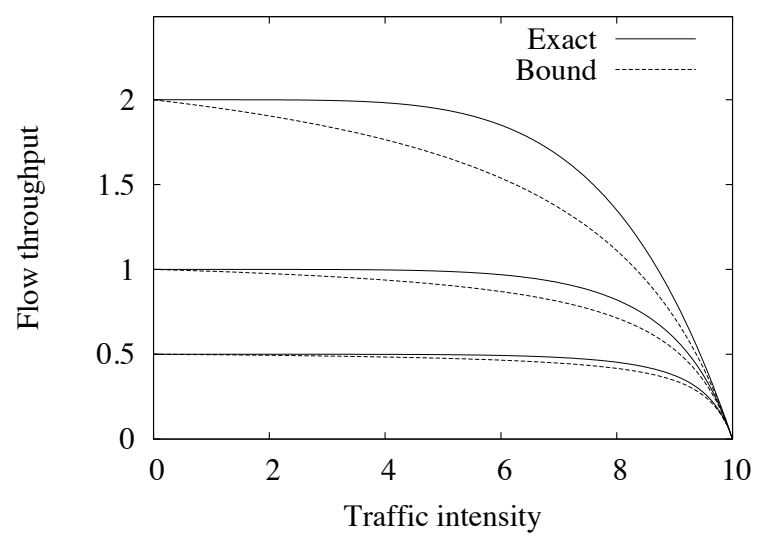

Fig. 8. Flow throughput for a wireline link with different fbw rate limits (classes 1,2,3, from top to bottom).

\section{CONCLUSION}

A key design objective of traffic control schemes in communication networks is to ensure maximum stability. The issue of throughput performance within the stability region is rarely addressed, however, except under the misleading assumption of a static number of flows. In practice, the number of competing flows is dynamic, which has a strong impact on throughput performance. The steady state distribution indeed determines the typical number of flows sharing the network resources and thus the mean bit rate of each of them. 
Balanced fairness has proved instrumental in evaluating this steady state distribution, which is intractable for usual allocations like max-min fairness and proportional fairness. Throughput performance can then be numerically evaluated by means of a simple recursive algorithm. The state space explosion limits the scope of such a numerical solution, however. The contribution of this paper is to provide simple, explicit bounds on the flow throughput for any network whose capacity constraints are linear. We believe these bounds can serve as guidelines to develop simple, practical engineering rules for a number of communication networks.

\section{APPENDIX I \\ PROOF OF LEMMA 1}

The proof is by induction on the total number of flows $n \equiv|x|$. The property holds for $n=1$ since

$$
\Phi\left(e_{i}\right)=\frac{A_{i l}}{C_{l}} .
$$

Now assume it holds for $n=m$, for some $m \geq 1$. Let $x$ be any state such that $x_{i}>0$ and $n=m+1$. By the induction hypothesis, we have for any resource $r$ :

$$
\begin{aligned}
\sum_{j} \frac{A_{j l}}{C_{l}} \Phi\left(x-e_{j}\right) & \geq \sum_{j, k} \frac{A_{j l}}{C_{l}} \frac{A_{k r}}{C_{r}} \Phi\left(x-e_{j}-e_{k}\right) \\
& =\sum_{k} \frac{A_{k r}}{C_{r}} \Phi\left(x-e_{k}\right) .
\end{aligned}
$$

Since the inequality holds for all $r$, it follows from (5) that:

$$
\Phi(x)=\sum_{j} \frac{A_{j l}}{C_{l}} \Phi\left(x-e_{j}\right) .
$$

\section{APPENDIX II}

\section{PROOF OF LEMMA 2}

In view of Lemma I, the inequality is equivalent to:

$$
\tilde{\Phi}\left(x, e_{0}\right) \leq\left(\max _{l} \frac{A_{i l}}{C_{l}}\right) \tilde{\Phi}(x, 0)+\sum_{l} \sum_{j} \frac{A_{j l}}{C_{l}} \tilde{\Phi}\left(x-e_{j}, e_{l}\right) .
$$

The proof is by induction on the total number of flows $n \equiv|x|$. The property holds for $n=0$. Assume it holds for $n=m$ and let $x$ be any state such that $n=m+1$. We denote by $r$ a resource that is saturated in state $\left(x, e_{0}\right)$ :

$$
\tilde{\Phi}\left(x, e_{0}\right)=\frac{A_{i r}}{C_{r}} \tilde{\Phi}(x, 0)+\sum_{k} \frac{A_{k r}}{C_{r}} \tilde{\Phi}\left(x-e_{k}, e_{0}\right) .
$$

By the induction hypothesis, we have:

$$
\begin{aligned}
\tilde{\Phi}\left(x, e_{0}\right) & \leq \frac{A_{i r}}{C_{r}} \tilde{\Phi}(x, 0) \\
& +\left(\max _{l} \frac{A_{i l}}{C_{l}}\right) \sum_{k} \frac{A_{k r}}{C_{r}} \tilde{\Phi}\left(x-e_{k}, 0\right) \\
& +\sum_{l} \sum_{j, k} \frac{A_{k r}}{C_{r}} \frac{A_{j l}}{C_{l}} \tilde{\Phi}\left(x-e_{j}-e_{k}, e_{l}\right) .
\end{aligned}
$$

Now it follows from (10) that for any class $j$ :

$$
\forall l \neq r, \quad \sum_{k} \frac{A_{k r}}{C_{r}} \tilde{\Phi}\left(x-e_{j}-e_{k}, e_{l}\right) \leq \tilde{\Phi}\left(x-e_{j}, e_{l}\right),
$$

and

$\frac{A_{i r}}{C_{r}} \tilde{\Phi}\left(x-e_{j}, 0\right)+\sum_{k} \frac{A_{k r}}{C_{r}} \tilde{\Phi}\left(x-e_{j}-e_{k}, e_{r}\right) \leq \tilde{\Phi}\left(x-e_{j}, e_{r}\right)$.

We deduce that:

$$
\begin{aligned}
\tilde{\Phi}\left(x, e_{0}\right) & \leq \frac{A_{i r}}{C_{r}} \tilde{\Phi}(x, 0)+\left(\max _{l} \frac{A_{i l}}{C_{l}}\right) \sum_{k} \frac{A_{k r}}{C_{r}} \tilde{\Phi}\left(x-e_{k}, 0\right) \\
& +\sum_{l} \sum_{j} \frac{A_{j l}}{C_{l}} \tilde{\Phi}\left(x-e_{j}, e_{l}\right) \\
& -\sum_{j} \frac{A_{j r}}{C_{r}} \frac{A_{i r}}{C_{r}} \tilde{\Phi}\left(x-e_{j}, 0\right) .
\end{aligned}
$$

Thus the proof will be completed if we show that:

$$
\begin{aligned}
\frac{A_{i r}}{C_{r}} \tilde{\Phi}(x, 0) & +\left(\max _{l} \frac{A_{i l}}{C_{l}}\right) \sum_{k} \frac{A_{k r}}{C_{r}} \tilde{\Phi}\left(x-e_{k}, 0\right) \\
& -\sum_{j} \frac{A_{j r}}{C_{r}} \frac{A_{i r}}{C_{r}} \tilde{\Phi}\left(x-e_{j}, 0\right) \\
& \leq\left(\max _{l} \frac{A_{i l}}{C_{l}}\right) \tilde{\Phi}(x, 0) .
\end{aligned}
$$

But this inequality may also be written:

$$
\left(\max _{l} \frac{A_{i l}}{C_{l}}-\frac{A_{i r}}{C_{r}}\right)\left(\tilde{\Phi}(x, 0)-\sum_{j} \frac{A_{j r}}{C_{r}} \tilde{\Phi}\left(x-e_{j}, 0\right)\right) \geq 0,
$$

which is satisfied in view of (10).

\section{REFERENCES}

[1] F. P. Kelly. Charging and rate control for elastic traffic. European Trans. on Telecommunications, 8: 33-37, 1997.

[2] M. Xiao, N.B. Shroff, E.K.P. Chong. A utility-based power-control scheme in wireless cellular systems. IEEE/ACM Trans. on Networking, 11: 210-221, 2003.

[3] F. Paganini, Z. Wang, J.C. Doyle, S.H. Low Congestion control for high performance, stability and fairness in general networks. IEEE/ACM Trans. on Networking, 13: 43-56, 2005.

[4] H. Han, S. Shakkottai, C.V. Hollot, R. Srikant and D. Towsley. Overlay TCP for multi-path routing and congestion control. Submitted to IEEE/ACM Trans. on Networking, 2006.

[5] G. de Veciana, T. J. Lee, and T. Konstantopoulos. Stability and performance analysis of networks supporting elastic services. IEEE/ACM Trans. on Networking, 1: 2-14, 2001.

[6] T. Bonald and L. Massoulié. Impact of fairness on Internet performance. In Proc. ACM Sigmetrics / Performance, 2001.

[7] H. Q. Ye. Stability of data networks under an optimization-based bandwidth allocation. IEEE Trans. Aut. Control, 48: 1238-1242, 2003.

[8] T. Bonald, L. Massoulié, A. Proutière and J. Virtamo. A queueing analysis of max-min fairness, proportional fairness and balanced fairness. To appear in Queueing Systems, 2006.

[9] T. Bonald, A. Proutière. Insensitive bandwidth sharing. IEEE Globecom, 2002.

[10] V. Paxson and S. Floyd. Diffi culties in Simulating the Internet. IEEE/ACM Trans. on Networking, 9: 392-403, 2001.

[11] T. Bonald and A. Proutière. On performance bounds for balanced fairness. Performance Evaluation, 55: 25-50, 2004. 